Pract. oto-rhino-laryng. 1960;22:150

\title{
Obliteration of the Frontal Sinus
}

\begin{tabular}{|l|l|}
\hline H.C. & Overbosch \\
\hline A.A. & Rap \\
\hline
\end{tabular}

From the Department of Otolaryngology, Lichtenberg-hospital, Amersfoort

Authors' address: H. C. Overbosch, Utrechtseweg 92. Amersfoort (Netherlands)

It may be impossible to establish the conditions for permanent healing in chronic frontal sinusitis. Several methods have been described to obliterate the frontal sinus; inlay of the frontal skin, inplantation of abdominal fat, bone chips and other materials have been successfully used. The authors used cancellous bone from the iliac crest. In three patients a complete cure was obtained. A coronal incision, $1 \frac{1}{8}$ inches behind the hairline, was made from ear to ear. The frontal skin was then turned downward and an excellent view of the region of the frontal sinuses was thus obtained. A thorough excision of the diseased sinus was carried out. It is essential to take away the whole anterior wall of the frontal sinus. Then the sinus was packed with little pieces of cancellous bone from the iliac crest.

The operation is easily performed, gives an optimal view of the operative region, yields good results and leaves no visible scar.

The authors stress the fact that they only operate when other conservative and operative measures have been exhausted. 